\title{
A cautious return to the conference circuit
}

\author{
Technology breakthroughs at the 2021 IEEE International Electron Devices Meeting, which is once again being held
} as an in-person event in San Francisco.

ack in July (Nat. Electron. 4, 449; 2021), we noted that conference organizers were cautiously exploring a return to in-person events, following a sustained period of virtual events due to the coronavirus pandemic. The emergence of the Omicron variant has quickly soured any optimistic thoughts of a smooth return to in-person conferences and provided a reminder - if any reminder was needed that the path out of the pandemic will be long and will require global solutions.

But conferences continue as best they can and one annual conference planning to return to an in-person event, following a year of being online only, is the IEEE International Electron Devices Meeting (IEDM). The event takes place in San Francisco this month and the research itself remains as strong as ever. In 2018, 2019 and 2020, we highlighted some of the breakthroughs reported at the meeting, and this year we return again to IEDM and offer our highlights of the 2021 event.

We begin with work on organic thin-film transistors, where Chen Jiang and colleagues at the University of Cambridge, Tsinghua University and the Suzhou Institute of Biomedical Engineering and Technology show how electronic fabrics can be created with organic transistors that are fabricated on plastic strips and then wrapped around stretchable fibres. As Simone Fabiano and Antonio Facchetti of Linköping University and Northwestern University explain in a News \& Views article about the work, the strip-helix-fibre architecture could prove useful in the development of unconventional optoelectronic and bioelectronic products.

Also at IEDM 2021, Jianshi Tang, Huaqiang $\mathrm{Wu}$ and colleagues at Tsinghua University and the Suzhou Institute of Nano-Tech and Nano-Bionics report on the three-dimensional (3D) integration of silicon complementary metal-oxide-semiconductor (CMOS) logic, compute-in-memory and associative memory, creating a system capable of implementing one-shot learning. Such machine learning requires only one (or a few) training samples, rather than the usual large datasets, and as Kai $\mathrm{Ni}$ and colleagues at the Rochester Institute of Technology explain in a News \& Views article, the 3D integration provides an important step toward creating a versatile system for information processing.

The potential role of $2 \mathrm{D}$ materials in the future of electronic circuits was the focus of last month's issue. In our third highlight from IEDM 2021, we return again to the topic with work from Kevin O'Brien, Chelsey Dorow and colleagues at Intel Corporation, which is discussed in a News \& Views article from Du Xiang and Tao Liu at Fudan University. The Intel team show, in particular, how transistors made from monolayer transition metal dichalcogenides can be fabricated on $300 \mathrm{~mm}$ wafers using an approach that is compatible with the back-end-of-line process temperatures involved in integrated circuit fabrication.

Elsewhere we highlight advances in medium-voltage power electronics and developments in millimetre-wave and terahertz technologies. We highlight work on creating ferroelectric random-access memory with antiferroelectric capacitors and work on building quantum dot image sensors with pixel sizes down to a pitch of $1.62 \mu \mathrm{m}$. Finally, we highlight progress in constructing photon-counting image sensors for low-light imaging and in using buried power lines for technology nodes beyond $3 \mathrm{~nm}$.

Published online: 10 December 2021 https://doi.org/10.1038/s41928-021-00704-8

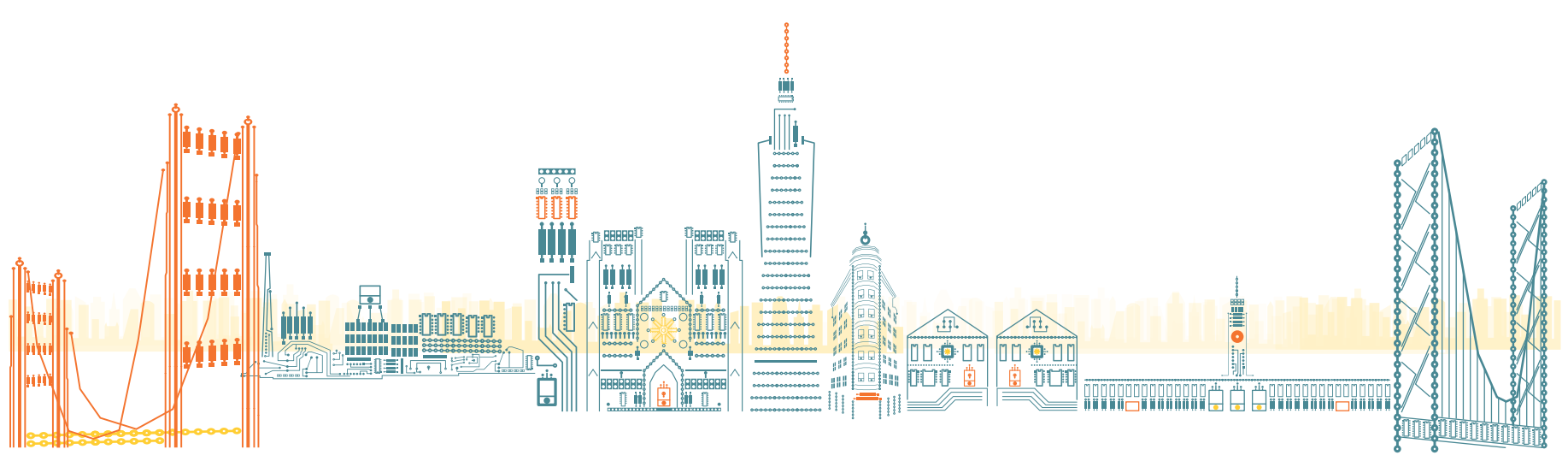

